Publications of the Astronomical Society of the Pacific, 114:1252-1259, 2002 November

(C) 2002. The Astronomical Society of the Pacific. All rights reserved. Printed in U.S.A.

\title{
Athermalizing Refractive Optics with Fluid Lenses
}

\author{
HARLAND W. EPPS \\ UCO/Lick Observatory, University of California, Santa Cruz, CA 95064; epps@ucolick.org \\ AND \\ DANiel G. FABricant \\ Harvard-Smithsonian Center for Astrophysics, 60 Garden Street, Cambridge, MA 02138; dfabricant@cfa.harvard.edu \\ Received 2002 June 9; accepted 2002 July 12; published 2002 October 1
}

\begin{abstract}
Astronomical optics may encounter a wide range of nighttime temperatures $\left(\sim-10^{\circ} \mathrm{C}\right.$ to $\left.+20^{\circ} \mathrm{C}\right)$ at mountaintop observatories. Complex refractive optics used in high-performance spectrographs and focal reducers may perform poorly at temperature extremes unless special care is taken in their design. Refocusing will not always restore image quality, and thermally induced focal length changes may introduce troublesome image motion. We describe the techniques that we have used to predict the thermal behavior of the Binospec Spectrograph, an instrument under development for the converted Multiple Mirror Telescope. This thermal analysis must account for (1) the change in optical power due to thermal expansion of the optical elements, (2) thermal variations in the refractive indices of the optical elements, and (3) element respacings due to the interplay between the thermal expansions of the optical elements and cell materials. Binospec's multiplets are fluid coupled to reduce the reflection losses that would occur at glass-air surfaces; to athermalize the Binospec optics, we form weak lenses in the optical coupling fluid within multiplets. The large variation of the coupling fluid's refractive index with temperature allows these weak fluid lenses to correct thermal changes in the optical elements and cell. This athermalization technique is attractive because it introduces no additional mechanical complexity.
\end{abstract}

\section{INTRODUCTION}

The wide range of temperatures encountered at mountaintop observatories must be addressed in the design of astronomical optics. Figure 1 shows the annual temperature variations ${ }^{1}$ at Mount Hopkins, Arizona, the site of the converted Multiple Mirror Telescope (MMT). Nighttime temperatures at the MMT span $\sim-10^{\circ} \mathrm{C}$ to $+20^{\circ} \mathrm{C}$. Even ideally supported and stressfree optics may fail to perform satisfactorily over such a wide temperature range. Refractive optics have three important thermally sensitive properties: (1) surface curvatures (dependent on the thermal expansion of the optical elements), (2) refractive indices, and (3) interelement spacings and lens thicknesses (dependent on the thermal expansion of the cell and optical elements). These thermal issues were important in the optical design of Binospec, a wide-field optical spectrograph under development for the MMT. In this paper, we describe how we athermalized the Binospec optics using coupling-fluid lenses. ${ }^{2}$

Some type of coupling medium (transparent adhesive, optical grease, oil, or other fluid) is frequently used in complex refractive systems to reduce the Fresnel reflection losses in-

\footnotetext{
${ }^{1}$ The data in Fig. 1 were compiled by Alejandra Milone, Carol Heller, and John McAfee at the MMT Observatory.

${ }^{2}$ Rogers \& Roberts (1995) review conventional active and passive athermalization techniques for a variety of optical systems.
}

curred by glass-air surfaces. For steeply curved optics larger than about $150 \mathrm{~mm}$, a fluid coupling is usually the only practical choice when materials having dissimilar thermal expansions are joined. A coupling-fluid lens is formed when the glass (or crystal) surfaces on the two sides of the coupling-fluid gap are not concentric. Coupling fluids have a strongly thermally dependent refractive index $n(T)$. For these liquids, $|d n / d T|$ is typically 100 times larger than for optical glasses. The thermal sensitivity of coupling fluids is usually an inconvenient feature. We have discovered that the large $|d n / d T|$ can be turned to advantage by forming weak positive or negative fluid lenses that compensate for the thermal behavior of the conventional optical elements and their cells. Since the coupling fluid may already be part of the system, the thermal compensation comes at no cost in mechanical complexity.

The use of liquid optical elements is reviewed briefly by Robb (1993), who found references to liquid lenses in the patent literature dating to 1924. Robb and his collaborators at Lockheed Palo Alto Research Laboratory and at Vavilov State Optical Institute in Russia published a series of papers describing the use of liquids to form optical elements (e.g., Sigler 1991, 1993; Robb, Tolstoy, \& Petrova 1993; Chow et al. 1993; Petrova et al. 1994a, 1994b; Fuller et al. 1996). These authors promoted liquid optical elements as an inexpensive replacement for abnormal-dispersion optical glasses or calcium fluoride in 


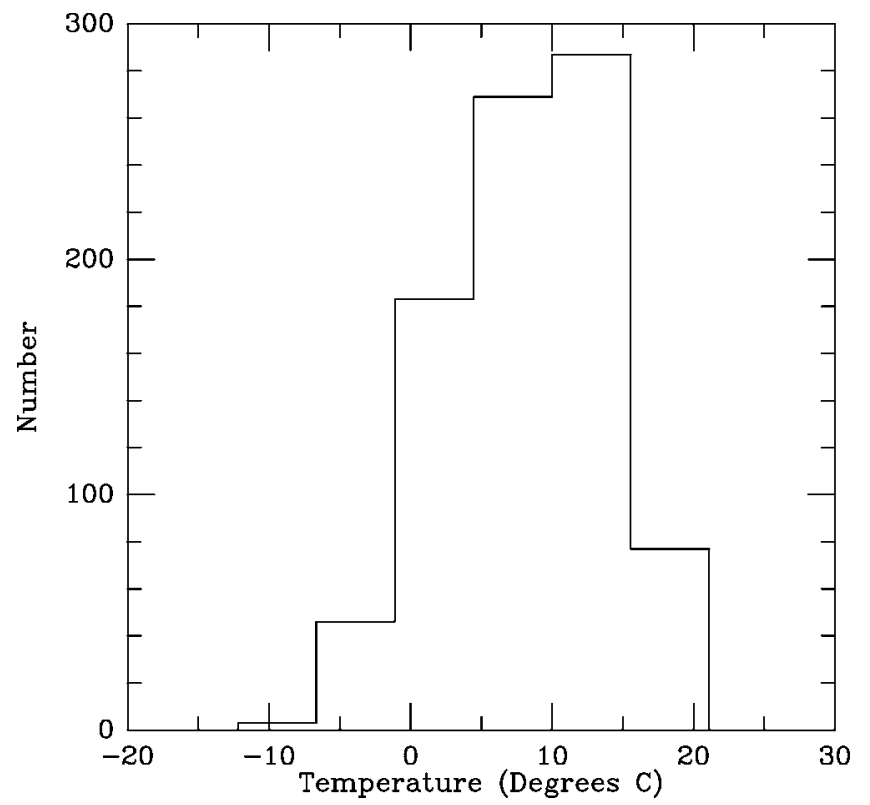

FIG. 1.- Histogram of the nighttime temperatures at the MMT on Mount Hopkins. The temperature was recorded during a seeing test performed on a large fraction of the nights that the telescope was in operation between 1991 and 1997. The data from 1995 were not included because of a seasonal bias. The mean temperature is $7.8^{\circ} \mathrm{C}$, with a standard deviation of $5.6^{\circ} \mathrm{C}$. These data were taken from Milone, Heller, \& McAfee (2000).

apochromatic photographic lenses and telescope objectives. Sigler (1990) lays out this design approach and describes an apochromatic telescope objective with three inexpensive glass elements (BK7 and F2) and two fluid lenses that is approximately athermal over a $\pm 15^{\circ} \mathrm{C}$ temperature range. Sigler describes how the potential thermal sensitivity introduced by the liquid lenses can be mitigated by the use of two fluids and appropriate design.

Our goals for coupling-fluid lenses differ from the Lockheed/ Vavilov group's: we use the coupling-fluid lenses to maintain the high performance of a complex refractive system over a considerable temperature range. Our optics contain calcium fluoride $\left(\mathrm{CaF}_{2}\right)$ and premium glass elements to address the largest possible field of view and to correct chromatic aberrations over a very wide band $(390-1000 \mathrm{~nm})$.

This paper is organized in the following fashion: $\S 2$ describes the performance of the initial design of the Binospec optics, $\S 3$ introduces our approach to athermal optical design with coupling-fluid lenses, $\S 4$ discusses our thermal analysis techniques, $\S 5$ summarizes the performance of the athermal Binospec design, and $\S 6$ presents our conclusions.

\section{THERMAL PERFORMANCE OF INITIAL BINOSPEC OPTICAL DESIGN}

The Binospec is a wide-field, moderate-dispersion optical spectrograph designed to be used at the $f / 5$ focus of the converted (6.5 m) MMT (Fabricant, Fata, \& Epps 1998). It will

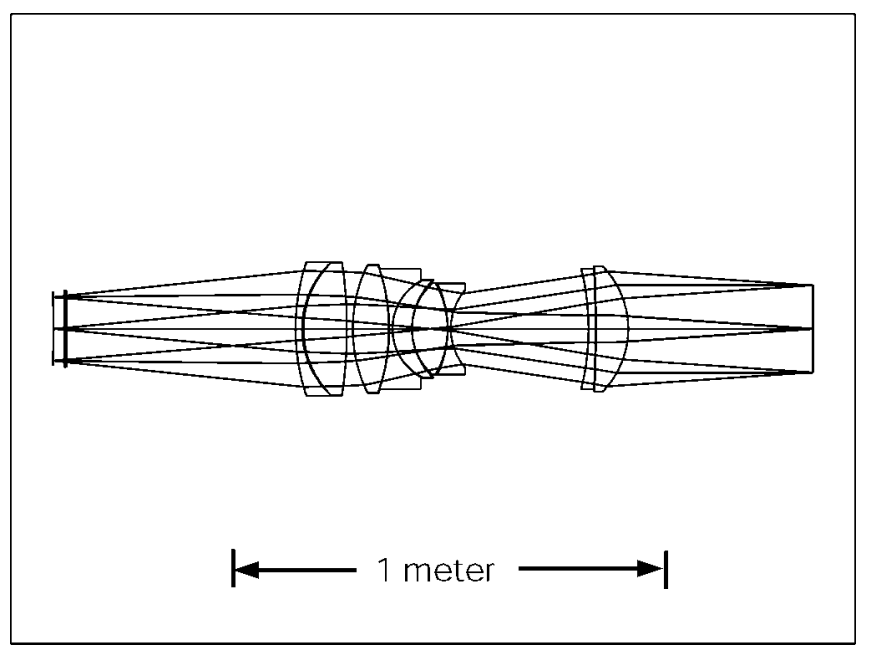

FIG. 2.-Optical layout of the Binospec collimator, with fold mirrors removed. From left to right, the optical materials are BAL15Y, S-FSL5Y, PBM2Y, PBL6Y, BAL35Y, $\mathrm{CaF}_{2}$, PBL6Y, BSM51Y, and $\mathrm{CaF}_{2}$. The telescope focal surface is on the left and the exit pupil is on the right.

use aperture masks with multiple slitlets to perform multiobject spectroscopy of faint galaxies. Binospec has two identical sets of refractive optics that address adjacent $8^{\prime} \times 15^{\prime}$ fields of view. Each set consists of a collimator (nine elements in three groups), a grating, and a camera (10 elements in four groups). The multiplets are coupled with Cargille Laser Liquid 5610. The collimator and camera together contain six $\mathrm{CaF}_{2}$ elements, and the camera contains an $\mathrm{NaCl}$ element. Figures 2 and 3 show the optical layout of the collimator and camera, respectively.

Because the image-quality requirements are very high, and because our spectroscopy is very sensitive to scale changes at

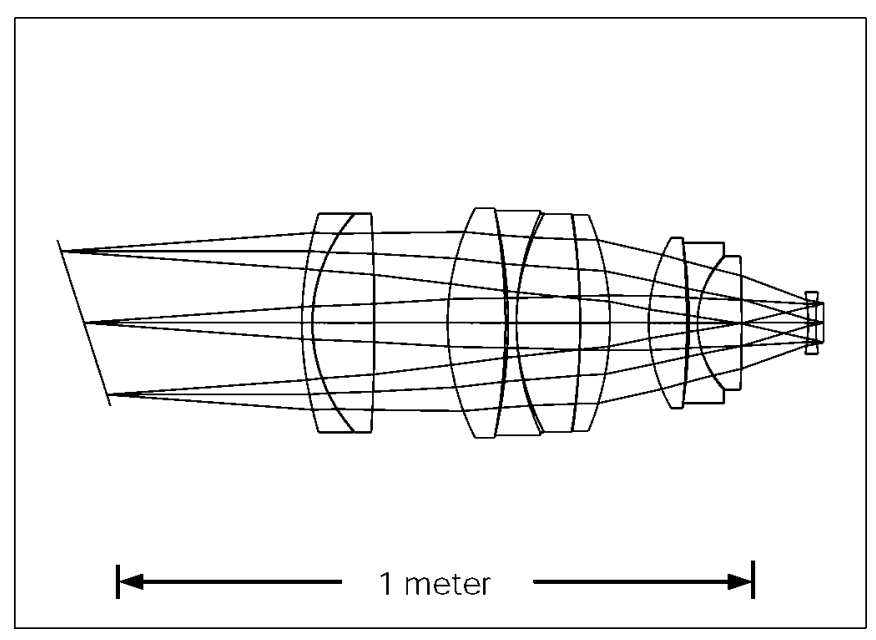

FIG. 3.-Optical layout of the Binospec camera. The last singlet serves as the Dewar window. From left to right, the optical materials are BAL35Y, $\mathrm{CaF}_{2}$, $\mathrm{CaF}_{2}$, BAL35Y, $\mathrm{CaF}_{2}$, PBM2Y, FPL51Y, NaCl, $\mathrm{CaF}_{2}$, and BSM51Y. 
TABLE 1

Thermal Coefficients for $\mathrm{CaF}_{2}$ AND $\mathrm{NaCl}$

\begin{tabular}{lrr}
\hline \hline Coefficient & $\mathrm{CaF}_{2}$ & $\mathrm{NaCl}$ \\
\hline$D_{0} \ldots \ldots \ldots$ & $-3.28552 \times 10^{-5}$ & $-8.71556 \times 10^{-5}$ \\
$D_{1} \ldots \ldots \ldots$ & $-3.38706 \times 10^{-8}$ & $-3.14215 \times 10^{-8}$ \\
$D_{2} \ldots \ldots \ldots$ & $2.34847 \times 10^{-10}$ & $-7.09548 \times 10^{-11}$ \\
$E_{0} \ldots \ldots \ldots$ & $4.76592 \times 10^{-7}$ & $1.69628 \times 10^{-6}$ \\
$E_{1} \ldots \ldots \ldots$ & $1.73774 \times 10^{-9}$ & $2.08920 \times 10^{-9}$ \\
$\lambda_{T K} \ldots \ldots \ldots$ & 0.13 & 0.16 \\
\hline \hline
\end{tabular}

the detector, we were concerned about the temperature stability of the Binospec optics. Our initial investigations revealed that this concern was well founded. Binospec's camera is fairly closely related to previous designs by Harland Epps (Epps 1998). Here, as in previous designs, a passive respace of a single lens group, coupled with a refocus of the detector, is sufficient to maintain excellent image quality and constant image scale over a wide temperature range. Refocus and respace motions of $\sim 5-10 \mu \mathrm{m}{ }^{\circ} \mathrm{C}^{-1}$ are required, depending on the chosen lens group. Unfortunately, this straightforward technique fails badly for Binospec's collimator. In order to maintain acceptable image quality and constant image scale over a wide temperature range, all three lens groups must be moved. The required movements are large, exceeding $150 \mu \mathrm{m}^{\circ} \mathrm{C}^{-1}$ for two lens groups. Very precise active control of three separate lens groups is required, a rather unappealing and rather risky prospect. Without thermal compensation, image shifts due to thermal changes in the collimator were of the order of 1 pixel ${ }^{\circ} \mathrm{C}^{-1}$ at the edge of the field, and image quality becomes unacceptable. Clearly, an athermal design is a necessity.

\section{THERMAL COMPENSATION WITH COUPLING-FLUID LENSES}

\subsection{Coupling-Fluid Properties Turned to Advantage}

Our approach to athermalization of Binospec's collimator (and camera) rests upon the fact that while the thermo-optical coefficients $(d n / d T)$ for most optical glasses are positive numbers of the order of a few parts per million, the corresponding numbers for typical optical coupling fluids are negative and perhaps 100 times larger in magnitude. Thus, it would seem plausible that if the coupling interfaces were allowed to become relatively low power fluid lenses, they would have substantial impact on the system's thermal behavior but would otherwise have little impact upon the optical design. These fluid lenses potentially offer sufficient degrees of freedom to allow passive athermalization while achieving image quality and lateral color correction comparable to a conventional optical design operating only at its design temperature.

In order to proceed with an optical design using fluid lenses, it is necessary to make some assumptions about the nature of the thermal perturbations and the lens cell design. We assume that while the collimator and camera lens assemblies may undergo a substantial temperature change during operation, they
TABLE 2

REFRACTIVE INDEX FOR LL5610, Lot 011673

\begin{tabular}{|c|c|c|c|c|c|c|}
\hline $\begin{array}{c}\lambda \\
(\mu \mathrm{m})\end{array}$ & $-15^{\circ} \mathrm{C}$ & $-10^{\circ} \mathrm{C}$ & $0^{\circ} \mathrm{C}$ & $8^{\circ} \mathrm{C}$ & $16^{\circ} \mathrm{C}$ & $25^{\circ} \mathrm{C}$ \\
\hline 0.435835 & 1.53487 & 1.53274 & 1.52858 & 1.52536 & 1.52212 & 1.51849 \\
\hline 0.479992 & 1.52714 & 1.52510 & 1.52101 & 1.51785 & 1.51466 & 1.51108 \\
\hline 0.546075 & 1.51941 & 1.51740 & 1.51740 & 1.51026 & 1.50712 & 1.50358 \\
\hline 0.587562 & 1.51599 & 1.51401 & 1.51003 & 1.50687 & 1.50374 & 1.50022 \\
\hline 0.643847 & 1.51239 & 1.51041 & 1.50642 & 1.50333 & 1.50024 & 1.49674 \\
\hline 1.013980 & 1.50193 & 1.49998 & 1.49611 & 1.49305 & 1.49001 & 1.48659 \\
\hline
\end{tabular}

Note.-Measurements by Ohara, Inc., using Carl Zeiss Jena Model PR-2 refractometer, V-block method. Measurement accuracy $\sim \pm 0.0001$, and temperature control to $\pm 0.1^{\circ} \mathrm{C}$.

always remain effectively isothermal. In any case, it is unlikely that any temperature gradient is likely to be stable enough that a more detailed model would be helpful. In the Binospec lens cell design, each lens element is mounted within a metal bezel using a room-temperature vulcanized (RTV) elastomer to fill the gap between the lens and the bezel. The thickness of the RTV elastomer is chosen to athermalize the differential thermal expansion of the lens and bezel. The aluminum bezels are registered to each other with an aluminum structure. The axial location of each lens element is then determined by the midplane of its aluminum bezel. As the temperature changes, it is a straightforward matter to determine the changes in the lens spacings.

\subsection{Multiple-Configuration Thermal Design}

Optical design optimization proceeds by minimizing a demerit function. The demerit function usually combines several terms: for example, image quality, wavelength coverage, field angle, path-length errors over the pupil, physical constraints, amplitudes of aspheric deviations, pupil location, distortion, etc. The construction of the demerit function (assigning appropriate weights to each term) and its application to find an "optimal solution" is the essence of computer-aided optical design. H. W. E.'s proprietary optical design code is named Optical Aberration Reduction and System Analysis (OARSA).

Conceptually, the implementation of the athermalization process into OARSA is straightforward. The process begins by computing the demerit function at a nominal operating temperature. The system is then thermally perturbed to the maximum and minimum design temperatures in turn, and the demerit functions are recalculated. The demerit functions at the three design temperatures are added in quadrature, with appropriate weighting functions. OARSA's predictive formalism determines the strategy for continuing with the optimization. The demerit function includes penalties for focus and lateral magnification changes with temperature, so as to drive the optimized solution toward an athermalized state with acceptable optical quality over the full temperature range.

Each optimization run proceeds until the multitemperature demerit function reaches a local minimum. For each run, the rms image diameters averaged over all field angles and wave- 


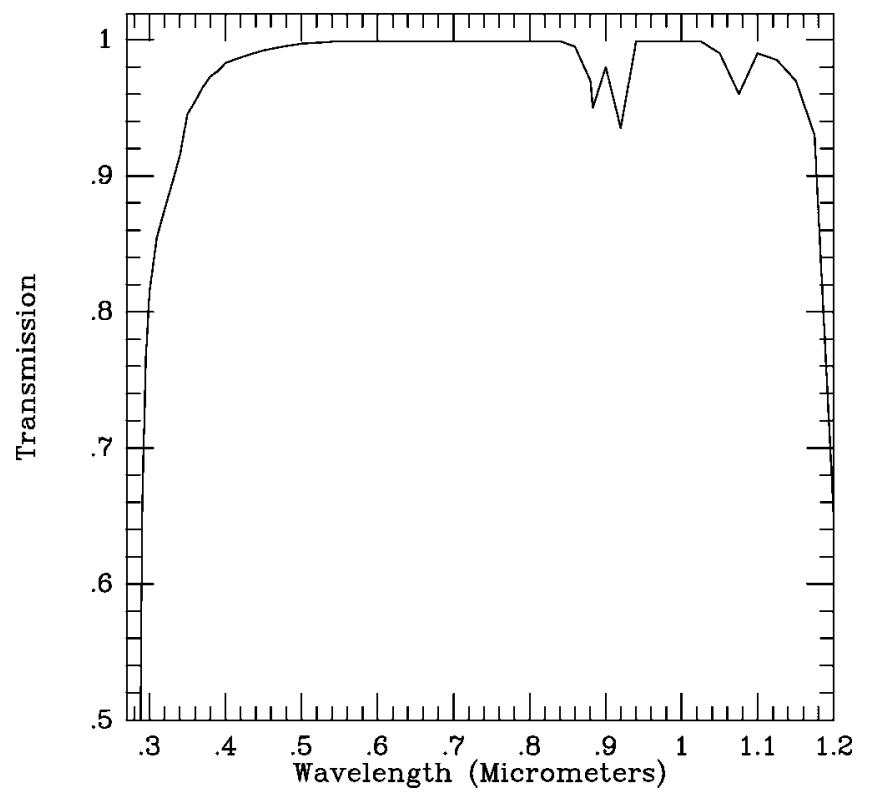

FIG. 4.-Internal transmission of Cargille LL5610 for a $1 \mathrm{~cm}$ path. The total path in Binospec's collimator and camera is approximately $1 \mathrm{~cm}$.

lengths (without refocus) and the maximum rms lateral color are calculated for the final optical design at the nominal operating temperature. In addition, the expected changes in focus and lateral magnification between the extremum and nominal design temperatures are calculated.

When it appears that an attractive solution has been found, more detailed ray tracing at multiple temperatures with the full Binospec optical model, using nine different grating configurations, confirms that the desired image and degree of athermalization has been attained. In practice, that detailed evaluation has been carried out by D. G. F. with the ZEMAX optical design and evaluation code. The transformations to different temperatures are made using a preprocessing code developed by D. G. F. This procedure thus provides a completely independent assessment of the Binospec optical design.

\section{THERMAL ANALYSIS OF BINOSPEC OPTICS}

\subsection{Thermal Modeling}

To make sure that we understand the assumptions and approximations that are part of the thermal model, we prefer a manual approach for analysis. We have analyzed the Binospec design at three temperatures: low $\left(-10^{\circ} \mathrm{C}\right)$, nominal $\left(8^{\circ} \mathrm{C}\right)$, and high $\left(25^{\circ} \mathrm{C}\right)$. We calculate glass catalogs appropriate for each of these temperatures and calculate the radii, lens thicknesses, and lens spacings using the appropriate coefficients of thermal expansion. If necessary, we can use finite-element modeling to predict the mechanical behavior of complex systems. If aspherics are present, as they are in the Binospec optics, their coefficients must be scaled appropriately: conic coefficients are dimensionless and aspheric coefficients have units of $r^{1-a}$,
TABLE 3

REFRACTIVE INDEX FOR LL5610, LoT 091475

\begin{tabular}{cccccc}
\hline \hline $\begin{array}{c}\lambda \\
(\mu \mathrm{m})\end{array}$ & $-4^{\circ} \mathrm{C}$ & $2^{\circ} \mathrm{C}$ & $8^{\circ} \mathrm{C}$ & $14^{\circ} \mathrm{C}$ & $20^{\circ} \mathrm{C}$ \\
\hline $0.365015 \ldots \ldots$ & & & & & 1.54032 \\
$0.404656 \ldots \ldots$ & 1.53748 & 1.53493 & 1.53246 & 1.53004 & 1.52756 \\
$0.435835 \ldots \ldots$ & 1.53027 & 1.52776 & 1.52530 & 1.52287 & 1.52042 \\
$0.486133 \ldots \ldots$ & 1.52184 & 1.51939 & 1.51694 & 1.51456 & 1.51211 \\
$0.546075 \ldots \ldots$ & 1.51505 & 1.51263 & 1.51023 & 1.50786 & 1.50547 \\
$0.587562 \ldots \ldots$ & 1.51164 & 1.50921 & 1.50681 & 1.50450 & 1.50210 \\
$0.656273 \ldots \ldots$ & 1.50740 & 1.50499 & 1.50262 & 1.50032 & 1.49795 \\
$0.706519 \ldots \ldots$ & 1.50510 & 1.50270 & 1.50037 & 1.49806 & 1.49572 \\
$0.852110 \ldots \ldots$ & 1.50062 & 1.49823 & 1.49592 & 1.49364 & 1.49131 \\
$1.013980 \ldots \ldots$ & 1.49767 & 1.49534 & 1.49300 & 1.49073 & 1.48843 \\
$1.128640 \ldots \ldots$ & & & & & 1.48700 \\
\hline \hline
\end{tabular}

Note. - Measurements by Ohara, Inc. Measurement accuracy $\sim \pm 0.0001$, and temperature control to $\pm 0.1^{\circ} \mathrm{C}$. Data provided by Brian Sutin of Carnegie Observatories.

where $r$ is the lens unit (mm, etc.) and $a$ is the order of the aspheric term.

\subsection{Refractive Index Variation With Temperature}

Optical glass manufacturers recognize the importance of measuring the thermal dependence of the refractive indices of their glasses, and they usually provide $d n / d T$ for several wavelengths over a $-40^{\circ} \mathrm{C}$ to $+80^{\circ} \mathrm{C}$ temperature range. These may be given as $d n_{\text {abs }} / d T$, values in a vacuum, or as $d n_{\text {rel }} / d T$, values with respect to air in thermal equilibrium. Since

$$
n_{\mathrm{abs}}=n_{\mathrm{air}} n_{\mathrm{rel}}
$$

it follows that

$$
\frac{d n_{\mathrm{abs}}}{d T}=n_{\mathrm{air}} \frac{d n_{\mathrm{rel}}}{d T}+n_{\mathrm{rel}} \frac{d n_{\mathrm{air}}}{d T}
$$

Since $n_{\text {air }} \sim 1$, this can be simplified to

$$
\frac{d n_{\mathrm{abs}}}{d T}=\frac{d n_{\mathrm{rel}}}{d T}+n_{\mathrm{rel}} \frac{d n_{\mathrm{air}}}{d T} .
$$

Perhaps the safest procedure is to work with absolute indices for the glass and convert to relative indices at the desired air temperature and pressure. ZEMAX has adopted the Schott Glass Technologies formula for the absolute glass indices and will calculate the glass index at the appropriate temperature if the appropriate coefficients are provided:

$$
\Delta n_{\mathrm{abs}}=\frac{n^{2}-1}{2 n}\left[D_{0} \Delta T+D_{1} \Delta T^{2}+D_{2} \Delta T^{3}+\frac{E_{0} \Delta T+E_{1} \Delta T^{2}}{\lambda^{2}-\lambda_{T K}^{2}}\right] .
$$

Here $\Delta T$ is the change in temperature referred to $20^{\circ} \mathrm{C}$ and $n$ is the relative index at standard temperature and pressure. The 
TABLE 4

Comparison of Original and Athermalized Binospec Optics

\begin{tabular}{|c|c|c|c|c|c|c|c|c|c|c|}
\hline \multirow[b]{3}{*}{ Design } & \multirow{3}{*}{$\begin{array}{c}\text { Temperature } \\
\left({ }^{\circ} \mathrm{C}\right)\end{array}$} & \multicolumn{9}{|c|}{$\begin{array}{l}\text { Average RMS Image Diameters } \\
\qquad(\mu \mathrm{m})\end{array}$} \\
\hline & & \multicolumn{9}{|c|}{ Configuration } \\
\hline & & 1 & 2 & 3 & 4 & 5 & 6 & 7 & 8 & 9 \\
\hline Original $\ldots . . .$. & 2 & 16 & 18 & 26 & 25 & 34 & 26 & 45 & 14 & 17 \\
\hline Original $\ldots . . .$. & 20 & 12 & 13 & 13 & 11 & 15 & 15 & 13 & 11 & 14 \\
\hline Athermal ...... & -10 & 13 & 17 & 12 & 11 & 15 & 16 & 13 & 12 & 14 \\
\hline Athermal $\ldots . .$. & 8 & 11 & 14 & 11 & 11 & 14 & 15 & 11 & 12 & 13 \\
\hline Athermal ...... & 25 & 13 & 15 & 12 & 13 & 14 & 15 & 12 & 13 & 14 \\
\hline
\end{tabular}

Note.-Configurations are described in Table 5. The original system was designed at $20^{\circ} \mathrm{C}$ and is not athermal. The loss of image quality and changes in lateral magnification of this system at low temperatures motivated the athermal design.

six coefficients $D_{0}, D_{1}, D_{2}, E_{0}, E_{1}$, and $\lambda_{T K}$ are available from the Schott catalog but must be calculated from the $d n / d T$ data provided by other manufacturers. This fit is in principle nonlinear, but an iterative linear fit using specified values of $\lambda_{T K}$ works well.

For $\mathrm{NaCl}$, we have found the most convincing $d n / d T$ data in Feldman et al. (1978) and in the 1902 data of F. J. Micheli, summarized in $\mathrm{Li}$ (1976). For $\mathrm{CaF}_{2}$, we use data of F. J. Micheli, summarized in Li (1980), Malitson (1963), and recent measurements by Ohara, kindly provided by B. Sutin (2001, private communication). We provide coefficients from our fits to the Schott thermal formula for $\mathrm{CaF}_{2}$ and $\mathrm{NaCl}$ in Table 1 .

In practice, D. G. F. uses precalculated catalogs of refractive indices at discrete temperatures in his analysis. He uses the Schott thermal formalism to check the independent calculations of H. W. E. that are based on corrections to $n_{\text {rel }}$ using $d n_{\text {rel }} / d T$.

\subsection{Coefficient of Thermal Expansion}

The coefficients of thermal expansion for optical glasses are given in the glass catalogs. Feldman et al. (1978) gives expansion coefficients for common optical crystals. The expansion coefficients for common cell materials are given in many references, but we urge caution since there are many errors in published values, and the temperature range of validity may have been lost in repeated transcriptions. Particular caution is appropriate for low-expansion alloys such as invar since the coefficients depend on the heat-treating history of the material. If coefficients of thermal expansion are in doubt, samples can be measured at a reasonable price.

Automatic calculations of interelement spacings as a function of temperature may not be handled correctly by optical design and analysis codes because they depend on the details of the lens mounting scheme. ZEMAX, for example, assumes that the cell material sets the spacing at the edge of the lenses, a good approximation in some but not all cases. In particular, for thick lenses effectively mounted at the midplane of the lens girdle (as appropriate for Binospec), ZEMAX's assumption is a poor approximation. Therefore, D. G. F. has written a separate code to move the element spacings to different temperatures and to create the appropriate ZEMAX file. H. W. E. has added code to OARSA to calculate the appropriate spacings.

\subsection{Cargille Laser Liquid 5610 Properties}

R. P. Cargille Laboratories are a prominent supplier of optical liquids that are used for a variety of applications. When

TABLE 5

Description of Binospec Optical Configurations

\begin{tabular}{|c|c|c|c|c|c|c|}
\hline \multirow{2}{*}{$\frac{\text { Configuration }}{1 \ldots \ldots \ldots \ldots}$} & \multirow{2}{*}{ 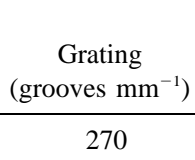 } & \multirow{2}{*}{$\begin{array}{c}\begin{array}{c}\text { Wavelength Range } \\
(\mu \mathrm{m})\end{array} \\
0.390-0.931\end{array}$} & \multicolumn{4}{|c|}{$\begin{array}{l}\text { Field Angles } \\
\qquad \begin{array}{c}(X, Y) \\
(\operatorname{arcmin})\end{array}\end{array}$} \\
\hline & & & $0.0,0.0$ & $0.0,3.8$ & $0.0,6.0$ & $0.0,7.5$ \\
\hline $2 \ldots \ldots \ldots$ & 650 & $0.390-0.620$ & $0.0,0.0$ & $0.0,3.8$ & $0.0,6.0$ & $0.0,7.5$ \\
\hline $3 \ldots \ldots \ldots$ & 650 & $0.766-1.000$ & $0.0,0.0$ & $0.0,3.8$ & $0.0,6.0$ & $0.0,7.5$ \\
\hline $4 \ldots \ldots \ldots$ & 900 & $0.572-0.741$ & $0.0,0.0$ & $0.0,3.8$ & $0.0,6.0$ & $0.0,7.5$ \\
\hline $5 \ldots \ldots \ldots$ & 900 & $0.831-1.000$ & $0.0,0.0$ & $0.0,3.8$ & $0.0,6.0$ & $0.0,7.5$ \\
\hline $6 \quad \ldots \ldots \ldots \ldots$ & 1200 & $0.390-0.516$ & $0.0,0.0$ & $0.0,3.8$ & $0.0,6.0$ & $0.0,7.5$ \\
\hline $7 \ldots \ldots \ldots$ & 1200 & $0.877-1.000$ & $0.0,0.0$ & $0.0,3.8$ & $0.0,6.0$ & $0.0,7.5$ \\
\hline $8 \ldots \ldots \ldots$ & Imaging & $0.390-0.500$ & $0.0,0.0$ & $2.0,3.8$ & $3.2,6.0$ & $4.0,7.5$ \\
\hline $9 \ldots \ldots \ldots$ & Imaging & $0.700-1.000$ & $0.0,0.0$ & $2.0,3.8$ & $3.2,6.0$ & $4.0,7.5$ \\
\hline
\end{tabular}

NotE. - Wavelength range is sampled at seven equally spaced intervals beginning at the shortest tabulated wavelength and ending at the longest. 
TABLE 6

Comparison of Original and Athermalized Binospec Optics

\begin{tabular}{|c|c|c|c|c|c|c|c|c|c|c|}
\hline \multirow[b]{3}{*}{ Design } & \multirow{3}{*}{$\begin{array}{l}\text { Temperature } \\
\left({ }^{\circ} \mathrm{C}\right)\end{array}$} & \multicolumn{9}{|c|}{$\begin{array}{c}\text { Average } 90 \% \text { EE Image Diameters } \\
(\mu \mathrm{m})\end{array}$} \\
\hline & & \multicolumn{9}{|c|}{ Configuration } \\
\hline & & 1 & 2 & 3 & 4 & 5 & 6 & 7 & 8 & 9 \\
\hline Original $\ldots . . . .$. & 2 & 24 & 26 & 39 & 35 & 50 & 37 & 62 & 20 & 25 \\
\hline Original $\ldots \ldots \ldots$ & 20 & 17 & 18 & 18 & 15 & 22 & 21 & 19 & 15 & 18 \\
\hline Athermal ...... & -10 & 18 & 24 & 16 & 15 & 21 & 23 & 17 & 17 & 17 \\
\hline Athermal ...... & 8 & 15 & 20 & 15 & 15 & 20 & 22 & 15 & 16 & 16 \\
\hline Athermal ....... & 25 & 19 & 20 & 16 & 17 & 19 & 20 & 16 & 17 & 19 \\
\hline
\end{tabular}

Note. - Configurations are described in Table 5. See also notes to Table 4.

we searched their catalog for an appropriate coupling fluid for the Binospec multiplets, we looked for liquids that transmit well between 390 and $1000 \mathrm{~nm}$, are well matched in index to the optical materials, are compatible with the cell materials (including the RTV elastomer seals), and remain liquid at temperatures above $-15^{\circ} \mathrm{C}$. We selected a siloxane fluid that Cargille designates Laser Liquid 5610. This LL5610 can be supplied with a $0.5893 \mu \mathrm{m}$ refractive index between $\sim 1.4750$ and $\sim 1.5340$ at $25^{\circ} \mathrm{C}$; we have chosen a formulation with an index of 1.500. The physical properties of LL5610 depend on the precise index formulation. In Figure 4, we plot the transmission of a $1 \mathrm{~cm}$ thickness of LL5610 (index $=1.5000$ ) as measured for us by Cargille Laboratories. Transmission of the coupling fluid is of particular concern for this athermalization scheme, since the total path through the coupling fluid is increased to form positive or negative lenses.

Precise measurements of the refractive index of LL5610 as a function of wavelength and temperature are essential for our application. These measurements were performed by Ohara, Inc. The results ${ }^{3}$ for two lots of LL5610 are given in Tables 2 and 3 . The refractive indices for the two different lots of fluid are consistent within the measurement errors.

One technique that we have used to interpolate the refractive index data in Table 2 or 3 to other wavelengths is to begin with a Schott dispersion formula fit to the $20^{\circ} \mathrm{C}$ data in Table 3:

$$
\begin{aligned}
n^{2}= & 2.19976744-2.50452328 \times 10^{-3} \times \lambda^{2} \\
& +1.81822150 \times 10^{-2} \times \lambda^{-2}+5.37568953 \times 10^{-4} \times \lambda^{-4} \\
& +4.47175840 \times 10^{-6} \times \lambda^{-6}+1.42223830 \times 10^{-6} \times \lambda^{-8}
\end{aligned}
$$

We fit a second-order polynominal in $1 / \lambda$ to the difference between the Schott formula and the measured indices and use this polynomial to interpolate between the measured indices. This is a procedure commonly used to apply "melt-sheet" corrections to the cataloged indices for optical glasses. The formalism of $\S 4.2$ can be used to interpolate indices at temperatures between those listed in Tables 2 and 3 .

\footnotetext{
3 The data in Table 3 were provided by Brian Sutin at Carnegie Observatories.
}

\section{PERFORMANCE OF ATHERMALIZED BINOSPEC OPTICS}

Tables 4 and 6 compare the rms image diameters and 90\% encircled energy (EE) image diameters of the original Binospec optical design (collimator and camera) at its design temperature of $20^{\circ} \mathrm{C}$ (and at $2^{\circ} \mathrm{C}$ ) with those of the athermalized optical design at three temperatures: $-10^{\circ} \mathrm{C}, 8^{\circ} \mathrm{C}$, and $25^{\circ} \mathrm{C}$. Table 5 summarizes the grating ruling densities, wavelength ranges, and field angles for the configurations presented in Tables 4 and 6 . While a temperature swing of $18^{\circ} \mathrm{C}$ severely compromises the performance of the original design, the athermal design suffers almost no loss of image quality over a $35^{\circ} \mathrm{C}$ temperature range. The worst-case image shifts between $-10^{\circ} \mathrm{C}$ and $25^{\circ} \mathrm{C}$ resulting from changes in lateral magnification are less than $4 \mu \mathrm{m}$, 2 orders of magnitude less than in the original design.

\subsection{Sensitivity of the Athermalized Design}

\subsubsection{The Effect of Temperature Gradients}

In a separate study (Brown, Fabricant, \& Boyd 2002), we have constructed a detailed thermal model of Binospec to predict the expected level of temperature gradients in the Binospec optics under extreme ambient conditions, when the dome temperature drops by over $17^{\circ} \mathrm{C}$ over the course of $24 \mathrm{hr}$. Temperature records show that a more typical daily swing in dome temperature is about $5^{\circ} \mathrm{C}$. We find that the radial and axial temperature gradients within lens groups are less than $0.2^{\circ} \mathrm{C}$ and $0.1^{\circ} \mathrm{C}$, respectively, even for the $17^{\circ} \mathrm{C}$ drop in dome temperature. The effect of these gradients is largely independent of our athermalization technique, but we have modeled them carefully.

A radial temperature gradient produces a radius of curvature change coupled with some spherical aberration. For a $0.2^{\circ} \mathrm{C}$ gradient, the radius of curvature changes (less than $0.5 \lambda$ peak to valley [PV]) are much smaller than our polishing specifications, while the worst-case spherical aberration is less than $\lambda / 6 \mathrm{PV}$, comparable to our stringent polishing specifications. The axial temperature gradients are even more benign, with comparable radius of curvature changes but negligible spherical aberration. (Here our reference is $0.6328 \mu \mathrm{m}$.) 
TABLE 7

EFFECT OF COUPLING-Fluid INDEX ERrors

\begin{tabular}{|c|c|c|c|c|c|c|c|c|c|c|}
\hline \multirow[b]{3}{*}{ Design } & \multirow{3}{*}{$\begin{array}{c}\text { Temperature } \\
\left({ }^{\circ} \mathrm{C}\right)\end{array}$} & \multicolumn{9}{|c|}{$\begin{array}{l}\text { Average RMS IMAGE Diameters } \\
\qquad(\mu \mathrm{m})\end{array}$} \\
\hline & & \multicolumn{9}{|c|}{ Configuration } \\
\hline & & 1 & 2 & 3 & 4 & 5 & 6 & 7 & 8 & 9 \\
\hline Athermal . & 8 & 11 & 14 & 11 & 11 & 14 & 15 & 11 & 12 & 13 \\
\hline Test A ......... & 8 & 11 & 14 & 12 & 11 & 15 & 15 & 13 & 10 & 12 \\
\hline Test $\mathrm{B} \ldots \ldots \ldots$ & 8 & 12 & 9 & 12 & 11 & 15 & 11 & 12 & 10 & 12 \\
\hline
\end{tabular}

Note. - In test A, the coupling-fluid refractive index was increased by 0.0005 independent of wavelength. In test $\mathrm{B}$, the index was increased by a term linear in wavelength (zero at $0.365 \mu \mathrm{m}$ and 0.0005 at $1.014 \mu \mathrm{m}$ ). The refractive index measurement error is 0.0001 .

The largest temperature gradients within the optics are $1^{\circ} \mathrm{C}$ under these extreme conditions. The second lens group in the collimator (a fluid-coupled quintet) and the second lens group in the camera (a fluid-coupled quartet) have temperatures lagging the less massive neighboring lens groups by up to $1^{\circ} \mathrm{C}$ (hotter in this case). We have modeled the situation with all of the optics at $8^{\circ} \mathrm{C}$, except for these two lens groups that are at $9^{\circ} \mathrm{C}$. The results are quite benign: image shifts resulting from changes in lateral magnification are less than $2 \mu \mathrm{m}$, and the image quality is indistinguishable from the isothermal system at $8^{\circ} \mathrm{C}$. Changes are quite slow as the thermal time constants for the optics exceed $40 \mathrm{hr}$.

\subsubsection{Required Accuracy of Coupling-Fluid Refractive Indices}

We have performed two numerical tests of the effects of errors in our knowledge of the index of refraction of the coupling fluid. The accuracy of the refractive index measurements in Tables 2 and 3 is \pm 0.0001 . In the first test, we added 0.0005 to the measured fluid indices, independent of wavelength. In the second, we added a term linear in wavelength that varied between zero at $0.365 \mu \mathrm{m}$ and 0.0005 at $1.014 \mu \mathrm{m}$. Table 7 compares the nominal $8^{\circ} \mathrm{C}$ performance with the performance using perturbed indices. We conclude that refractive index measurement errors of \pm 0.0001 are adequate.

\section{CONCLUSIONS}

Thermal analysis of the original optical design for Binospec, a wide-field optical spectrograph, revealed a troubling loss of image quality and large changes in lateral magnification over the temperature range encountered at the MMT Observatory. We have refined the original optical design after developing a new technique for athermalizing refractive optics. Our approach is to form weak lenses in the fluid used to couple the multiplet elements; the large variation of the coupling fluid's refractive index with temperature allows these weak fluid lenses to compensate for thermal changes elsewhere. The athermalization is therefore accomplished without additional mechanical complexity.

The performance of the athermalized system between $-10^{\circ} \mathrm{C}$ and $25^{\circ} \mathrm{C}$ is quite comparable to the performance of the original (thermally sensitive) system at its design temperature. We describe our thermal analysis and thermal design technique in sufficient detail to allow others to use our technique. We present measurements of the thermal dependence of the refractive index of the chosen coupling fluid (Cargille LL5610), as well as our fits to $d n / d t$ for $\mathrm{CaF}_{2}$ and $\mathrm{NaCl}$ from selected data in the literature. Finally, we demonstrate that our athermalized design is insensitive to the expected temperature gradients in the optics and to the current measurement errors in the refractive indices of the coupling fluid.

We thank Warren Brown and David Boyd for their work on the thermal analysis of Binospec discussed in $\S 5.1$. Robert Fata, Binospec's project engineer, has helped with many aspects of this analysis. Andrew Fanning carried out the early thermal analysis of Binospec and alerted us to the thermal problems with the original designs. Finally, we thank Brian Sutin for providing additional index data for the Cargille LL5610 and fits to these data.

\section{REFERENCES}

Brown, W., Fabricant, D., \& Boyd, D. 2002, PASP, in press

Chow, A. W., Leary, D. F., Sigler, R. D., Robb, P. N., Petrova, M. V., \& Tolstoy, M. N. 1993, Proc. SPIE, 2018, 206

Epps, H. W. 1998, Proc. SPIE, 3355, 111

Fabricant, D., Fata, R., \& Epps, H. 1998, Proc. SPIE, 3355, 232

Feldman, A., Horowitz, D., Waxler, R., \& Dodge, M. 1978, NBS

Tech. Note 993, Natl. Tech. Inf. Ser. PB292245 (Springfield: NBS)
Fuller, J. B., Robb, P. N., Sigler, R. D., Abramov, A. P., Petrova, M. V., Plyukhin, A. G., \& Tolstoy, M. N. 1996, Proc. SPIE, 2863, 442

Li, H. H. 1976, J. Phys. Chem. Ref. Data, 5, 329 1980, J. Phys. Chem. Ref. Data, 9, 161

Malitson, I. H. 1963, Appl. Opt., 2, 1103

Milone, A., Heller, C., \& McAfee, J. 2000, Meteorological Data for Mt. Hopkins (MMT Tech. Memo; Tucson: MMTO) 
Petrova, M. V., Plyukhin, A. G., Tolstoy, M. N., Robb, P. N., Sigler, R. D., \& Leary, D. 1994a, Proc. SPIE, 2263, 19 1994b, Proc. SPIE, 2263, 24

Robb, P. N. 1993, Proc. SPIE, 2018, 216

Robb, P. N., Tolstoy, M. N., \& Petrova, M. V. 1993, Proc. SPIE, 2018, 200
Rogers, P. J., \& Roberts, M. 1995, in Handbook of Optics, ed. M. Bass et al. (2d ed.; New York: McGraw-Hill), chap. 39 Sigler, R. D. 1990, Appl. Opt., 29, 2451

1991, Proc. SPIE, 1535, 89

1993, Proc. SPIE, 2018, 188 